\title{
Kernos
}

Revue internationale et pluridisciplinaire de religion grecque antique

$10 \mid 1997$

Varia

\section{Le dernier roi d'Athènes, entre le mythe et le rite}

\section{Marco V. García Quintela}

URL : http://journals.openedition.org/kernos/653

DOI : $10.4000 /$ kernos. 653

ISSN : 2034-7871

Éditeur

Centre international d'étude de la religion grecque antique

Édition imprimée

Date de publication : 1 janvier 1997

Pagination : 135-151

ISSN : 0776-3824

Référence électronique

Marco V. García Quintela, «Le dernier roi d'Athènes, entre le mythe et le rite », Kernos [En ligne],

10 | 1997, mis en ligne le 12 avril 2011, consulté le 21 avril 2019. URL : http://journals.openedition.org/ kernos/653; DOI : 10.4000/kernos.653 


\title{
Le dermier roi d'Athènes :
}

\author{
entre le mythe et le rite
}

Je vais analyser ici comment se présente un fait vécu par un personnage athénien nommé Hippoménès selon diverses versions. Mais je dois faire avant tout une remarque sur le titre : j'annonce qu'il s'agit du dernier roi d'Athènes; en réalité, c'est une inexactitude qui a pour but d'éviter un autre titre excessivement long. Plus précisément, Hippoménès était le dernier des Médontides, une famille qui a occupé pendant de longues années la plus haute magistrature athénienne: royauté ou archontat décennal selon les différentes sources.

Les Médontides constituent une famille curieuse qui a été l'objet de nombreuses approches de la part des chercheurs. Il s'agit de la deuxième dynastie royale athénienne. Elle succède aux Théséides, appelés aussi Érechthéides, qui étaient autochtones et à propos desquels nous connaissons un bon nombre de mythes dans diverses versions. C'est justement le caractère marginal de cette deuxième dynastie dans les traditions, en opposition à la première, qui a mené à l'existence d'un éventail d'interprétations de la part des modernes. Ainsi, sa présence serait due au besoin qu'avaient les Atthidographes de remplir le vide chronologique existant entre le dernier Érechthéide et les premiers archontes. Les rares épisodes qui se réfèrent à ses membres sont aussi l'objet des appréciations les plus variées quant à leur ancienneté, leur fiabilité historique ou leur caractère mythique. De plus, comme le démontre F. Jacoby, la tradition qui précise la localisation institutionnelle des Codrides ou Médontides (les deux noms sont en usage pour faire référence à cette dynastie : le premier vient du deuxième de ses membres et le second, du troisième) se divise en deux tendances. La première propose une évolution institutionnelle simple : elle considère les Médontides comme des rois qui étaient au pouvoir jusqu'à ce que les archontes apparaissent en l'an 683. La seconde expose une reconstruction plus compliquée : Médon était déjà archonte à vie comme ses successeurs qui, à partir d'une certaine date, n'auraient plus occupé la magistrature que pour dix ans. En tout cas, les deux traditions présentent Hippoménès comme le dernier des Médontides au pouvoir et le considèrent en tant que roi pour la première et 
archonte pour la seconde ${ }^{1}$. Elles coïncident aussi sur les circonstances étranges au cours desquelles Hippoménès a perdu la magistrature.

Je présenterai tout d'abord les diverses versions de cet épisode pour établir ensuite qu'il s'agit d'un mythe que l'on peut analyser en tant que tel. Ceci me conduira à une étude comparative qui montre que ce mythe a été fabriqué à partir d'une altération du sens du sacrifice du cheval tel que nous en avons connaissance chez d'autres peuples indo-européens. J'examinerai enfin les témoignages de l'existence historique en Grèce de sacrifices de chevaux.

\section{L'histoire du dernier Médontide}

Ce que nous savons sur Hippoménès se réduit à l'épisode qui raconte comment il perd le pouvoir. Cet épisode se trouve dans plusieurs fragments de caractère historique ou d'allusions à un toponyme de l'Attique dont la relation est établie clairement grâce au thème qui leur est commun et au nom des personnages qui y interviennent. Nous partirons d'un fragment aujourd'hui disparu de la Constitution des Atbéniens d'Aristote, fragment qui nous servira de référence pour le reste des autres versions :

On n'élisait plus de rois parmi les Codrides car on considérait qu'ils étaient devenus efféminés et mous ( voulut se démarquer de cette accusation. Ayant surpris un amant ( $\mu$ ol $\chi o ́ s)$ avec sa fille Leimônè, il fit mourir le premier en l'attachant à son char et enferma sa fille avec un cheval jusqu'à ce qu'elle meure (Aristt., Ath. fr. $1=$ fr. 385 Rose).

Aristote suit ici la reconstruction la plus simple, Hippoménès est roi; il recueille ensuite, dans le même texte $(3,3)$, une version plus compliquée de la tradition, mieux représentée dans le texte de Nicolas de Damas, dans lequel Hippoménès est un archonte :

...il a perdu le pouvoir pour la raison suivante : il avait une fille qui avait été déshonorée en secret par un habitant de la cité; mû par la colère il l'enferma dans une étable en l'attachant à un cheval et ne leur fit pas mander de nourriture à aucun des deux. Et ainsi, le cheval se trouvant subjugué par la faim, après avoir attaqué la jeune fille et l'avoir mangée, mourut aussi en fin de compte. Après ces

\footnotetext{
1 L'étude systématique de ces traditions a été réalisée par F. JACOBY dans son édition des FGrHist, A Commentary on the Ancient Historians of Atbens Illb, Leiden, 1954, p. 43-51 dans le commentaire du fr. 23 d'Hellanicos de Lesbos, les analyses de P. CARLIER, La royauté en Grèce avant Alexandre, Strasbourg, 1984, p. 360-9 et de R. DREWS, Basileus. The Evidence for Kingship in Geometric Greece, New Haven - London, 1983, p. 86-94 acceptent Jacoby; Drews recueille l'historiographie sur la crédibilité de cette liste royale. P. BARCELÓ, Basileia, Monarchia, Tyrannis, Stuttgart, 1993, p. 39 n. 103, ne s'arrête pas sur ce cas.
} 
faits et une fois que la maison eut été détruite par Hippoménès lui-même, le lieu fut connu sous le nom du Cheval et la Jeune Fille ${ }^{2}$.

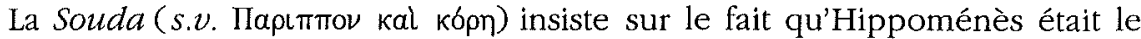
dernier archonte des Codrides et ajoute un jeu de mots significatif à propos de son nom : il indique qu'Hippoménès enferma sa fille «avec un cheval fou » pour détruire sa virginité et explique aussi le nom du lieu à partir de l'épisode. La version d'Eschine est un peu différente : elle se présente comme un simple aition qui explique le nom du lieu et est en relation avec ses intérêts immédiats, invoquant la constitution ancestrale des Athéniens. Dans ce récit, le père offensé est un simple citoyen ( $\pi 0 \lambda\left(\tau \epsilon_{S}\right.$ ) qui découvre la faute de sa fille et la punit comme nous l'avons vu en l'enfermant avec un cheval dans un endroit dénommé «Le Cheval et la Jeune Fille »; il poursuit en présentant la loi de Solon à propos des femmes adultères comme si elle émanait du même esprit rigoureux et louable des ancêtres ${ }^{3}$.

En définitive, les différentes versions de cet épisode présentent des lignes de force communes, mais elles comportent des détails qui diffèrent d'une version à l'autre, ce qui permet de reconstruire une séquence relativement complète: Hippoménès, le dernier Codride qui a exercé le pouvoir à Athènes (en tant que roi ou archonte décennal) et que l'on jugeait trop mou et peu viril, a voulu rétablir son honneur en intervenant dans la situation de sa famille. Pour cela, il punit sa fille, Leimônè, et un homme qui commettait l'adultère avec elle; mais il ne suit pas le procédé punitif établi, raison pour laquelle il aurait perdu le pouvoir. Le châtiment consiste à dételer l'un des chevaux de son char, atteler l'amant à sa place et enfermer ce cheval avec la jeune fille, sans vivres, jusqu'à ce que le cheval la dévore ou lui fasse perdre sa virginitét

2 FGrHist, $90 \mathrm{~F}$ 49. Presque la même chose dans la Souda, s.u. Hippoménès, et DIOD. SIC., VIII, 22; la source commune à tous ceux-ci serait Éphore, selon Jacoby.

3 Contre Timarque, 182-183; à propos de ce genre d'emploi de Solon, voir M.I. FINLEY, The Ancestral Constitution in The Use and Abuse of History, London, 1975, p. 34-59, et C. MOSSÉ, Comme s'élabore un mytbe politique: Solon, 'père fondateur' de la démocratie atbénienne, in Annales(ESC),

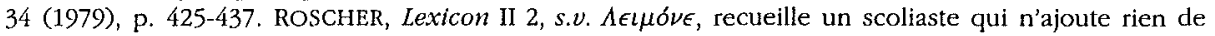
nouveau. DION CHR., 32, 78-79 considère que le lieu dénommé « Le Cheval et la Jeune Fille » est un sanctuaire.

4 Avec Mélanthos, le fondateur de la dynastie, et Codros, son successeur immédiat, Hippoménès est le troisième et le dernier des Médontides de qui il est dit quelque chose. Au sujet de Mélanthos, voir F.J. FERNÁNDEZ NIETO, Los acuerdos bélicos en la antigua Grecia, vol. II, Saint Jacques de Compostelle, 1975, p. 15-20, traitement historiciste et compilation de toutes les sources; P. VIDAL-NAQUET, El cazador Negro, Barcelone, 1983, p. 135-158 analyse son histoire comme un mythe. À propos de Codros, voir CARLIER, op. cit. (n. 1), p. 360-361. Selon JACOBY, op. cit. (n. 1), p. 50-51, ce récit est probablement «un aition authentique " employé par Hellanicos pour expliquer pourquoi les Athéniens ne choisissaient plus leurs rois parmi les Codrides et pourquoi, à leur place, ils choisissaient des archontes, tout d'abord décennaux puis annuels; pour DrEWS, op. cit. (n. 1), p. 92 , cet épisode serait le seul "truly mythical " de la série. Je ne partage pas le point de vue de Jacoby selon lequel Hellanicos compila des histoires libres pour les incorporer à sa reconstruction dynastique. Les épisodes mythiques des deux fondateurs et du dernier roi de la dynastie ont sans aucun doute un sens dont le dévoilement exigerait un travail à part. De toute manière ces 


\section{Analyse interne et caractère mythique de l'épisode}

Plusieurs questions appellent une première approche. Tout d'abord les noms des personnages sont significatifs, comme l'indique l'une des versions de la Souda. De plus, la situation familiale et sexuelle de Leimônè et les différents délits en question semblent confus. D'autre part, il faut préciser le rôle du cheval. Enfin, il est clair que le châtiment appliqué a un lien avec la perte du pouvoir, mais d'une manière qui ne trouve aucune explication dans les diverses versions à notre disposition.

\subsection{Les noms des personnages}

Il s'agit de noms qui parlent d'eux-mêmes. Celui de la fille ne présente pas de problèmes : $\Lambda \epsilon \iota \mu \hat{\nu} \eta$ est la prairie en tant qu'endroit humide qui est un régal pour les yeux à cause de sa splendeur; mais son usage comme métaphore du sexe féminin peut aussi être significatif ${ }^{5}$.

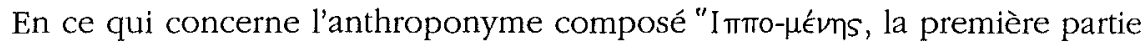
ne présente pas de difficultés car le mot $\imath \pi \pi$ os est fréquent dans les anthroponymes d'aristocrates dont il souligne le lien privilégié avec les chevaux. Mais, l'élément - $\mu$ évns, qui donne son sens complet au nom, n'a aucune référence directe bien qu'il existe divers termes proches du point de vue phonétique.

traitements analysent peu l'épisode qui nous intéresse plus spécialement; seul CARLIER, op. cit. (n. 1), p. 366, évoque le parallélisme entre le cas d'Hippoménès et l'búbris attribuée aux tyrans dont la tradition essaierait de rapprocher le dernier Codride. D'autres interprétations se concentrent sur le châtiment: pour G. GLOTZ, L'Ordalie dans la Grèce primitive, Paris, 1904, p. 100-101, l'épisode se situerait dans une série d'ordalies par emmurement des fitles coupables sur ordre de leur père; dans ce cas, l'esprit de l'ordalie se trouverait dans le refus d'exécuter la peine de mort soi-même et dans la décision de remettre son application à la volonté des dieux; G. HOFFMANN, Le châtiment des amants dans la Grèce classique, Paris, 1990, p. 40-42, s'occupe seulement de la version d'Eschine, qu'elle juge ancienne et présente comme un exemple approprié de ta sévérité possible dans ce genre de situation; T.J. SAUNDERS, Plato's Penal Code. Tradition, Controversy and Reform in Greek Penology, Oxford, 1991, p. 77-80 et 358 inclut notre cas parmi les châtiments pour des crimes spécifiques : il s'agit d'un châtiment "réflexif » qui consiste à convertir la faute commise en châtiment; ainsi l'offense se transforme en punition des amants par la substitution du cheval à l'adultère. D'un autre point de vue, G. DEVEREUX, Les chevaux antbropophages dans les mythes grecs, in REG, 88 (1975), p. 203-205, signale la confluence de deux faits distincts dans ce genre de récits; d'une part la réalité de l'existence de chevaux qui mangent des protéines animales et, d'autre part, la peur infantile, prouvée dans le domaine de la psychanalyse, du "cheval dévorant», symbole du père. P. GHIRONBISTAGNE, Le cheval et la jeune fille ou de la virginité chez les anciens Grecs, in Pallas, 32 (1985), p. 105-121 n'étudie pas tous les témoignages et ignore la question de la royauté, clef de notre hypothèse de travail. Aspect que ne considère pas non plus W.D. O'FlAHERTY, Women, Androgynes, and Other Mytbical Beasts, Chicago - London, 1980, p. 204, qui ne s'intéresse pas non plus à toutes les versions du mythe (p. 195).

5 EUR., Cycl., 168-172 avec le commentaire d'A. MOTTE, Prairies et jardins de la Grèce Ancienne, De la religion à la philosopbie, Bruxelles, 1973, p. 50-53. Voir aussi J. PIGEAUD, Euripide et la connaissance de soi. Quelques réflexions sur Hippolyte 73 à 82 et 373 à 430, in LEC, 44 (1976), p. 3-24, spéc. p. 4. 
En premier lieu, nous pouvons le relier à $\mu$ t́vos, le pouvoir ou l'énergie que les guerriers - dont l'épopée dessine une image positive - reçoivent des dieux. De plus il s'agit d'une qualité qui peut être attribuée aussi à des éléments naturels, comme le feu, et à des animaux, surtout aux chevaux. En tout cas, il s'agit d'un état physique anormal chez la personne qui l'obtient ${ }^{6}$. D'autre part, il peut être mis en rapport avec $\mu a v i a$, possibilité qu'évoque la Souda (s.v. Parippos kai koré) quand elle souligne la folie du cheval qui est l'instrument du châtiment. Dans ce cas, il faut aussi citer d'autres composés proches comme le verbe into$\mu a v e ́ \omega$ qui indique le désir des juments en chaleur d'être avec un étalon (Aristt., $H A, \mathrm{VI}, 18,572 \mathrm{a} 10)$ ou les noms $i \pi m o \mu a v l a$, qui indique la passion pour les chevaux, ou immouavís, qui fait référence à l'abondance de chevaux. Un composé spécial est le neutre iтrouavris qui peut faire allusion à une plante particulière que les chevaux aiment à la folie ou qui les rend fous (Théocr., II, 47 52; Théophr., $H P, \mathrm{XI}, 15,6$ ), ainsi qu'à une excroissance charnue des poulains nouveau-nés que leurs mères ont l'habitude de manger et que la magie grecque tenait pour un philtre puissant que l'on essayait d'obtenir par-dessus tout (Aristt., HA, VI, 22, 577a 7-14; VIII, 24, 605a 2-6; Élien, $N A$, III, 17; XIV, 17). Enfin, on appelait ainsi les pertes vaginales des juments en chaleur, que l'on considérait aussi comme un puissant aphrodisiaque ${ }^{7}$.

Quoi qu'il en soit, le nom évoque un lien avec les chevaux qui n'est pas normal, comme un cheval inspiré d'une force extraordinaire ou sentant une passion extrême pour les chevaux qui confine à la folie et qui évoque de manière négative, du point de vue d'un anthroponyme qui se veut sans aucun doute honorable, une forte pulsion sexuelle.

Pour finir, nous citerons un vers de Sophocle qui met en rapport les noms

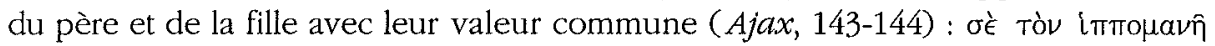

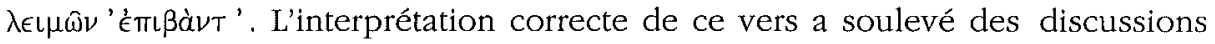

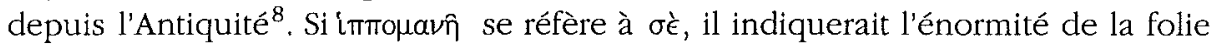
d'Ajax quand il tue des bêtes qui paissent dans la prairie. Si on le met en rapport avec $\lambda \epsilon \iota \mu \hat{\omega} \nu$, il qualifie la prairie et toute l'expression peut être interprétée comme «pré luxuriant » ou bien «pré où les chevaux sont abondants». Cette dernière possibilité indique une proximité forte entre les deux termes qui est, en fait, celle

6 HOM., Il., XVII, 456; XXIII, 468; voir E.R. DODDS, Los Griegos y lo Irracional, Madrid, 1980, p. 22-24; J.M. REDFIELD, Nature and Culture in the lliad. The tragedy of Hector, Chicago - London, 1975, p. 172 et sq.; M. DARAKI, Le béros à menos et le béros daimoni isos, une polarité bomérique, in

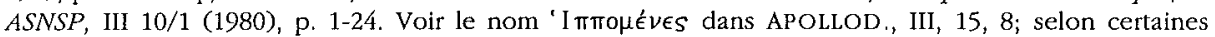

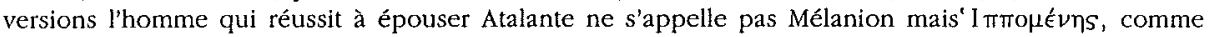

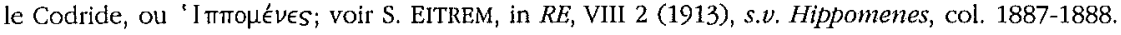

7 ARISTT., H.A., VI, 18; 572a 25-29, PAUS., V, 27, 3-4. A.M. TUPET, La magie dans la poésie latine. I. Des origines à la fin du règne d'Auguste, Paris, 1976, p. 79-81, souligne les fondements grecs des techniques; A. BERNAND, Sorciers grecs, Paris, 1991, s'intéresse au contexte social et mental de l'activité magique. Voir aussi F. GRAF, La magie dans l'antiquité gréco-romaine, Paris, 1994.

8 Fr. ELLENDT, Lexicon Sophocleum (Hildesheim, Zurich, New York 1986) (réimp.), s.v. Hippomanés, recueille diverses scolies, et voir Souda, s.v. Hippomanê. 
qui existe entre les personnages athéniens et qui est très troublante si nous tenons compte des résonances sexuelles de leurs noms.

\subsection{La situation familiale}

Le contexte familial que décrit notre histoire présente de nombreux paradoxes. Le roi, qui devrait être surtout un bon guerrier, viril par excellence (comme le furent les fondateurs de la dynastie qui se sont fait remarquer par leurs faits d'armes) est présenté comme un homme mou ( $\mu \alpha \lambda \alpha \kappa i a, \tau \rho v \phi n ́)$, qui, en tant qu'aristocrate et membre d'une société dominée par les principes de honte et d'honneur', doit récupérer une réputation perdue aux yeux de ses sujets. Pour cela, il doit intervenir dans la vie sexuelle de sa fille et c'est alors que surgissent les problèmes.

Tout d'abord, il s'agit sans aucun doute de sa fille. Mais l'homme qui maintient des relations sexuelles avec elle est présenté à différentes reprises comme rouxós. Or D. Cohen a indiqué récemment que, contre ce qui était établi, la

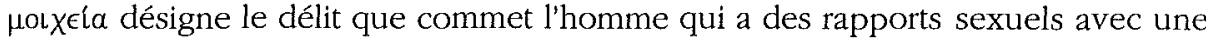
femme mariée; les femmes célibataires ou veuves qui ont des relations avec un homme libre, toujours du point de vue androcentrique, ne souffrent pas l'action d'un adultère, auquel on se réfère exclusivement par le terme $\mu$ o $\chi o ́ s$, mais elles sont l'objet de la séduction ou du rapt ${ }^{10}$. Dans ce sens la version que donne Eschine de cet épisode est significative, puisque cet auteur fait disparaître l'adultère en insistant sur la faute commise par la fille qui perd sa virginité avant le mariage. Cependant, il est clair que le thème de la $\mu$ oı $\chi \in l \alpha$ était latent quand il loue les normes de Solon contre les femmes adultères. Ceci conduit à trois possibilités.

La première : Leimônè était mariée avec un autre homme qui n'apparaît pas dans l'histoire. Si tel était le cas, son kúptos, c'est-à-dire l'homme qui agirait légalement en son nom et pourrait être touché par ses offenses ${ }^{11}$, serait probablement son mari et non son père. Dans ce cas, la faute d'Hippoménès serait d'être intervenu dans les compétences d'un oikos qui lui était étranger bien qu'ayant un lien avec lui. Cette hypothèse est la moins fiable puisqu'il n'y a aucune allu-

9 DODDS, op. cit. (n. 6), p. 39-70; A.W.H. ADKINS, Merit and Responsability, Oxford, 1960; D. COHEN, Law, Sexuality, and Society. The enforcement of morals in classical Atbens, Cambridge, 1991.

10 Ibid., p. 98-170; ID., The Athenian Law of Adultery, in RIDA, 31 (1984), p.147-65; ID., Sexuality, violence, and the Athenian Law of Hubris, in GR, 37 (1991), p. 171-188. Point de vue traditionnel dans U.E. PAOLI, Il reato di adulterio in diritto Attico, in SDHI, 16 (1950), p. 123-182; A.R.W. HARRISON, The Law of Atbens, I, The Family and Property, Oxford, 1968, p. 32-38; S.G. COLE, Greek Sanctions Against Sexual Assault, in CPb, 79 (1984), p. 97-113; E.M. HARRIS, Did the Atbenians regard seduction as a worse Crime than Rape?, in CQ, 40 (1990), p. 370-377.

11 HARRISON, op. cit. (n. 10), p. 30-32 et 108-115; W.K.LACEY, The Family in Classical Greece, London, 1968, p. 21-25. 
sion au mari et que le toponyme qui découle de cette histoire présente Leimônè

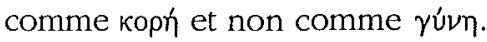

La seconde possibilité serait que le terme notxós est mal employé puisque la jeune fille n'est pas mariée. Une variante de cette hypothèse consisterait à recourir aux textes que nous avons vus pour aboutir à l'interprétation traditionnelle sur l'adultère dans le droit athénien comme un délit que peut commettre n'importe quelle femme intégrée dans le oikos d'un homme donné. Mais l'analyse que Cohen fait de ce sujet semble assez fiable et élimine cette possibilité; de

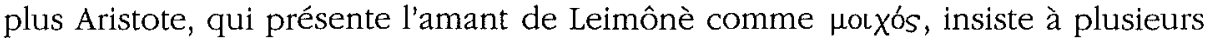
reprises sur la spécificité du délit dans des contextes matrimoniaux ${ }^{12}$.

La dernière possibilité localiserait l'erreur dans la description de la relation entre Hippoménès et Leimônè, qui ne seraient pas père et fille, mais mari et femme; l'amant de cette dernière serait effectivement un $\mu$ ot xós. Une telle hypothèse ne résiste pas à tous les témoignages qui présentent Leimônè comme

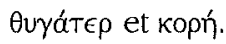

S'il n'y a pas d'erreur et si Leimônè est une jeune kopł́ dont l'amant est

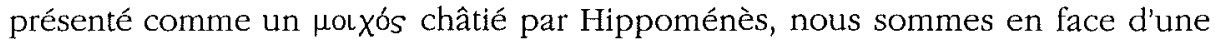
situation familiale atypique et inquiétante, comme celle que nous venons d'indiquer dans le paragraphe antérieur où nous signalions la proximité qu'évoquent les noms des deux personnages.

\subsection{Le rôle du cheval}

Le cheval et l'amant sont interchangeables, comme le souligne le châtiment qui voit le second remplacer le premier dans l'attelage d'Hippoménès et l'inverse auprès de Leimônè. De plus, il n'est pas besoin d'insister sur la proximité séman tique et conceptuelle entre le fait de manger et le rapport sexuel, proximité presque universelle et présente aussi en Grèce ${ }^{13}$. Notre histoire insiste sur ce trait quand il est dit que, les deux personnages enfermés ayant faim, le cheval dévore Leimônè ou, selon une autre version, lui fait perdre sa virginité, soulignant ainsi le rôle sexuel du cheval ${ }^{14}$. Tout ceci vient d'une tradition d'origine indo-

12 ARISTT., E.N., V, 6; 1134 a 19-21, souligne l'importance de savoir si la femme est mariée; ibid., $\mathrm{V}, 11,1138 \mathrm{a} 25-27$, on ne peut pas moicheúei avec sa propre femme; E.E, 1221b. Dans ARISTOPH., EC., 225, 520 (et contexte), moichós renvoie aux femmes mariées; au v. 911 et sq., une jeune femme qui n'est pas mariée n'appelle pas l'homme avec lequel elle a des rapports moichós.

13 Par exemple ARISTOPH., Acb., 764-834. E. LEACH, Aspectos antropológicos del lenguaje: categorias animales e injuria verbal, in E.H. LENNEBERG (ed.), Nuevas direcciones en el Estudio del Lenguaje, Madrid, 1974, p. 37-82, la p. 61 présente un tableau qui montre l'homologie entre la classification établie par les Anglais pour l'accessibilité sexuelle et la comestibilité de diverses espèces animales; A. SCHNAPP-GOURBEILLON, Lions, béros, masques, Paris, 1981, montre que les chevaux et les chiens sont les alter ego des héros d'Homère.

14 Souda, s.v. Parippo kai koré; voir G. SISSA, Le corps virginal. La virginité féminine en Grèce ancienne, Paris, 1987; J.M. GÓMEZ PALLARES, Del simbolisme eròtic de la imatge del cavall, in Faventia, 14 (1992), p. 7-33. La série abondante de parallèles recueillie par O'FLAHERTY, op. cit. (n. 4), p. 149-212, montre l'origine indo-européenne de cette relation. 
européenne qui insiste sur la proximité entre les deux espèces, dans le sacrifice par exemple, comme nous le verrons plus loin ${ }^{15}$.

Pour le moment nous pouvons signaler un détail intéressant pour notre histoire, détail dont la véracité ethnographique nous importe moins que le fait qu'il reflète des idées acceptables dans la pensée grecque. Selon Hippocrate, avant leur mariage, les femmes sauromates se comportaient comme des guerriers, entre autres choses elles montaient à cheval, ce qu'elles ne faisaient plus à partir du moment où elles se mariaient ( $A$ ër., 17); ainsi, pour les femmes, l'équivalence entre le cheval et le mari est manifeste. D'autre part, du point de vue masculin, un goût excessif pour l'équitation entraîne, comme inconvénient, l'impotence caractéristique de beaucoup d'hommes scythes (Aër., 22).

Dans la zoologie éthologique des Grecs, que l'on peut distinguer de l'éthologie anatomique par le fait qu'elle reçoit l'influence d'un grand éventail de croyances populaires et de mythes, en marge du caractère plus ou moins scientifique des auteurs qui la transmettent ${ }^{16}$, on peut suivre une «biographie» du cheval parallèle à celle de l'homme.

Tout d'abord, dès le moment de la procréation, les juments ressemblent aux femmes, car les unes comme les autres sont avides de plaisirs, raison pour laquelle on appelle juments les femmes qui ne peuvent pas mettre un frein à leurs rapports sexuels (Sémon., fr. 7 D., 57-70; Aristt., $H A$, VI, 18, 571b 8-13; Élien, $N A$, VI, 11). La femme et la jument se ressemblent aussi dans leur luxure puisqu'elles sont les seules femelles à avoir des rapports sexuels pendant la gestation (Aristt., HA, VII, 4, 585a 3-4; Élien, NA, VI, 11). Les juments se caractérisent aussi par leur coquetterie, leur goût pour les parfums et leur rejet des amants qui ne sont pas désirables à cause de leur laideur (Sémon., fr. 7 D., 63-66; Élien, NA, II, 10; XII, 16; XI, 24). Quand elles ont eu leur petit, elles se comportent tout à fait comme les femmes, elles se caractérisent par l'amour démesuré qu'elles vouent à leurs rejetons, amour provoqué par l'ingestion, juste après la naissance, de l'hippoménès que nous avons déjà mentionné. De plus, en cas de besoin, une jument qui n'est pas la mère d'un poulain donné peut en devenir la nourrice (Élien, NA, III, 17; VI, 48; XIV, 18; Aristt., HA, VIII, 24, 605a 2-7). D'autre part, les chevaux respectent le tabou de l'inceste, comme le montre l'histoire d'un roi scythe qui possédait une jument et un poulain dotés d'excellentes qualités. Comme ils n'avaient pas de partenaire, il les obligea à copuler à leur insu; quand ceux-ci s'aperçurent de ce qui s'était passé, ils se suicidèrent (Élien, $N A$, VI, 7). Plusieurs témoignages signalent la facilité avec laquelle les chevaux apprennent et mémorisent, et surtout leur passion pour la musique, qui les

15 J. PUHVEL, Aspects of Equine Functionality, in J. PUHVEL (éd.), Myth and Law Among the IndoEuropeans, Berkeley, 1970, p. 159-172; G. DUMÉZL, La Religion romaine archaïque, Paris, $1974^{2}$ p. 287-289; F. LE ROUX, Notes d'bistoire des Religions II, in Ogam, 7 (1955), p. 291-296.

16 M. VEGETTI, Los Origenes de la Racionalidad Cientifica. El Escalpelo y la Pluma, Barcelone, 1981; M. GARCía QUINTELA, L'anthropogonie d'Anaximandre. Problèmes d'interprétation tournant autour de la sagesse milésienne arcbaïque, in QS, 26 (1987), p. 161-176. 
stimule sexuellement, et leur habileté pour la danse (Élien, $N A$., XII, 44; XV, 25; XVI, 23 et 25). Les chevaux ressemblent physiquement à leurs propriétaires et peuvent recevoir un traitement médical inspiré par Sérapis comme le frère ou le fils de leur maître (Élien, $N A$, III, 2: XI, 31; $c f$. A ristt., $H A$, VIII, 24, 604b 25-27). À la fin de leurs jours, les chevaux exceptionnels peuvent recevoir des honneurs funèbres dignes de ceux des hommes (Hdt., VI, 103; Élien, NA, XII, 40).

Ce type de zoologie nous présente des cas de triangles amoureux avec des chevaux et des hommes qui sont pertinents ici. Dans un cas relaté par Élien, un garçon d'écurie, épris d'une jument jolie, «comme pourrait l'être une belle jeune fille », eut des rapports avec elle, mais celle-ci avait un poulain qui tua l'amant à cause de son despotisme; de plus, il fit subir des humiliations au cadavre enterré du zoophile ${ }^{17}$. Il faut aussi citer ici les juments de Diomède le Thrace, conquises par Héraclès dans son huitième travail et qui tuèrent Abdère, éromène du héros chargé de les surveiller (A pollod., II, 5, 3).

Il existe d'autres cas moins cruels où les chevaux sont mêlés aux relations érotiques; celui de l'Athénien Soclès, homme d'une grande gentillesse qui avait acheté un cheval «beau comme lui » ayant une grande intelligence et très ardent. L'attachement érotique du cheval pour son maître éveilla les soupçons des voisins à tel point que Soclès vendit le cheval qui se laissa mourir de faim (Élien, NA, VI, 44). Il faut aussi citer dans ce contexte la coutume originaire de Thessalie que signale Élien, sans en donner d'explications, selon laquelle pendant la noce le marié offre à la mariée un cheval avec tout son équipement ${ }^{18}$. Enfin rappelons que Zeus offre des chevaux à Tros, le père de Ganymède, en contrepartie du rapt de l'adolescent ${ }^{19}$.

Tout en tenant compte de la proximité existant entre les chevaux et leurs maîtres ${ }^{20}$, la passion sans ambiguité que les juments sentent pour leurs poulains

17 ÉL., N.A., IV, 8. Dans la lyrique, on trouve fréquemment la comparaison entre les jeunes qui inspirent le désir sexuel et les chevaux ou juments : ALCMAN, 1, 45-51 et 58-59 LGS; ANACR., 243, 304, 335 LGS; IBYC. 267 LGS; THÉOG., 257-260, 1249-1252, 1253-1254 (= SOLON, 13 D.), 1267-1270.

18 ÉL., N.A., XII, 34. Poséidon fait aussi cadeau de chevaux immortels à Pélée pour ses noces, APOLLOD., III, 13, 5; Polydectes demande des chevaux à ses amis pour les offrir à Hippodamie quand il l'épouse, ibid., II, 4; 2.

19 HOM., Il. V, 265; H. Ven., 210; PAUS, V, 24.5; APOLLOD., II, 5; 9. Le cheval est aussi important lors des noces d'Hélène: Tyndare exige que les prétendants de sa fille jurent sur un cheval sacrifié qu'ils appuieront celui qui obtiendra la main de la jeune fille, PAUS., III, 20, 9. Un autre sacrifice d'un cheval en rapport avec une faute sexuelle se trouve dans PLUT., Erotikai diegéseis, 3; une autre version dans ID., Pel, 20-22 où le sacrifice d'une jument substitue celui d'une vierge; il semble difficile de tirer un usage commun de toutes ces données si ce n'est la relation générique existant entre les humains et les chevaux, qui se réfère surtout à des thèmes érotiques; voir M. CAPOZZA, Spartaco e il sacrificio del Cavallo, in Critica Storica, 2 (1963), p. 251-293, et p. 273-282 sur des sacrifices de chevaux en Grèce avec bibliographie antérieure.

20 Une autre référence significative est l'explication de la tripartition de l'âme selon Platon : un aurige et deux chevaux aux caractéristiques différentes, précisément dans un contexte érotique, Phd., $253 \mathrm{c}-257 \mathrm{~b}$. 
et vice versa, ainsi que de l'importance de la dimension érotique de tous ces récits, revenons à notre histoire.

Les chevaux du char d'Hippoménès s'identifient à leur maître. Ainsi, quand il punit sa fille par l'intermédiaire du cheval, c'est comme s'il la punissait luimême. Mais l'amant, qui remplace le cheval dans l'attelage, s'identifie aussi au

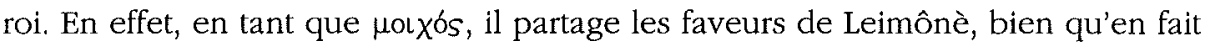
elle soit la fille d'Hippoménès. Enfin, le cheval enfermé avec la fille remplace l'amant qui au lieu de lui faire l'amour la mange, mais ce serait aussi le cadeau idéal pour une éventuelle noce qui se présente dans cet épisode sous des traits aberrants.

Pour conclure cette partie, nous évoquerons le mythe d'Hippodamie dont le père CEnomaos, roi de Pise, était épris. La jeune fille le rejetait, raison pour laquelle son père l'empêchait de se marier. En effet, il mettait les prétendants de sa fille au défi de fuir sur son char avec elle. Si le roi les atteignait, il tuait l'aurige, sinon il lui donnait sa fille. CEnomaos tua ainsi de nombreux prétendants jusqu'au jour où Pélops, grâce à une ruse d'Hippodamie, vainquit le roi, le tua et conquit son royaume ${ }^{21}$.

Si nous revenons à notre histoire en ayant ce mythe à l'esprit, les rapports entre nos personnages s'éclaircissent. La proximité du père et de la fille est due à une pulsion incestueuse (peu importe qu'elle ait été menée à bien ou pas), qui conduit à présenter l'amant comme rotxós. Si celui-ci pouvait institutionnaliser la relation qui existe entre les deux, il deviendrait un héritier possible du trône d'Athènes et pourrait offrir des chevaux à la mariée pour leurs noces. Mais l'intervention du père qui voudrait prendre, précisément, la place de l'amant détruit cette relation et provoque le crime double et cruel des amants.

21 APOLLOD., Ep. II, 3-9 et d'autres sources recueillies dans l'édition de la $L C L$ p. 156-163. Une comparaison plus approfondie entre l'histoire d'Hippoménès et le mythe d'Hippodamie nous mènerait trop loin. En effet, il faudrait inclure dans cette étude le parallèle presque exact qu'implique la relation entre Euénos, sa fille Marpessa et son prétendant Idas, ainsi que le cas d'Arès qui tue Halirrhothios parce qu'il a violé sa fille Alkippé et la situation qui se produit entre le violent Kerkyôn, sa fille Alopé et l'amant de celle-ci, Poséidon. Tous ces épisodes doivent être interprétés comme le partage de fonctions établi par la mythologie grecque entre Arès et Poséidon. Dans tous les cas que nous avons cités, Arès est du côté du père violent et possessif (il donne ses chevaux à Enomaos, il est le grand-père de Marpessa et le père d'Alkippé et, bien qu'il n'apparaisse pas dans le mythe d'Alopé, son caractère violent est proche de celui de Kerkyôn) et Poséidon du côté de l'amant (il donne ses chevaux vainqueurs à Idas et Pélops - dont il a aussi été éraste -, il est l'amant d'Alopé et le père d'Halirrhothios). Voir une approche de ces épisodes, insuffisante du point de vue que nous avons signalé, dans O'FLAHERTY, op. cit. (n. 4), p. 190-202, qui souligne aussi la racine indoeuropéenne de l'aspect incestueux de ces épisodes p. 167-171, 178, 183-185 et 195 avec une mention directe d'Hipppoménès. Au sujet de la mythologie de Poséidon, en mettant l'accent sur sa relation avec les chevaux, voir F. SCHACHERMEYR, Poseidon und die Entstebung des griechischen Götterglaubens, Munich, 1950, p. 22, 34 n., 37-38, et passim. 


\subsection{Le châtiment infligé}

Il ne s'agit pas d'une peine prévue par le droit athénien pour le rotxós, le séducteur ou l'auteur du rapt, ou pour la femme, mariée ou pas, qui aurait des rapports illicites. D'une part, il est rare que l'on donne la mort à l'adultère. Si elle est provoquée lorsqu'il est découvert en flagrant délit, ce serait là une circonstance atténuante ${ }^{22}$. Ce qui était correct, c'était de parvenir à un accord privé avec paiement d'une indemnisation. Si ce n'était pas le cas, le mari accusait l'amant devant les Onze qui pouvaient reconnaître la faute et l'affaire pouvait passer devant les tribunaux. Dans ce cas, si on le déclarait coupable, le rox xós pouvait subir des peines infamantes ${ }^{23}$. De toute façon, un accord économique était toujours possible. Quant à la femme, son mari était obligé de demander le divorce sous peine d'ảtula car, s'il ne le faisait pas, les hommes qui forçaient leurs femmes à se prostituer et/ou qui, de connivence avec elles, faisaient du chantage aux amants surpris pourraient agir impunément. De plus la femme mariée était privée du droit de participer à la vie religieuse de la cité et on pouvait la soumettre à diverses vexations qui n'aboutissaient jamais à la mort ${ }^{24}$. Tout cela n'a rien à voir avec la perte de la royauté qui est à l'origine de cet épisode. Et l'allusion à l'buibris d'Hippoménès n'est pas non plus une explication suffisante.

En définitive les ambiguïtés de noms parlants mais pas assez explicites, la situation familiale confuse avec l'évocation possible de l'inceste entre les personnages, la présence significative des chevaux et la nature non pénale, ou hors-la-loi, du châtiment nous conduisent à penser que nous nous trouvons devant un mythe ${ }^{25}$. Comme l'explique C. Lévi-Strauss «ce qui est propre à la pensée mythique, c'est de s'exprimer avec l'aide d'un répertoire dont la composition est hétéroclite et qui, bien qu'ample, n'est pas illimité : néanmoins il est indispensable qu'elle y puise, quelle que soit la tâche qu'elle s'impose, parce qu'elle n'en a aucun autre auquel elle puisse avoir recours ${ }^{26}$. Nous avons jusqu'ici fait l'inventaire de tous les éléments inclus dans notre histoire, à l'exception de celui qui donnera son sens à l'épisode entier.

22 DÉM., 23, 53-55. Dans LYSIAS, Sur le meurtre d'Eratóstenes, l'homicide d'un adultère est obligé de se défendre; COHEN, op. cit. (n. 1), p. $105-117$ et 131-132 signale l'idée de châtiments plus forts dans le passé.

23 ID., A Note on Aristophanes and the Punishment of Adultery in Athenian Law, in ZfR RömAbt., 102 (1985), p. 385-387, sceptique à propos de la qualité des sources sur ce sujet; HOFFMANN, op. cit. (n. 4), p. 33-35, traditionnel.

24 COHEN, op.cit. (n, 1), p. 98 et sq.

25 Déjà dans ce sens S. EITREM, très bref, dans RE, VIII 2 (1913), s.v. Hippomenes 3, col. 1888 et, plus récents, Jacoby et Drews cités à la n. 4.

26 Dans El Pensamiento Salvaje, México, 1964, p. 35-36. 


\section{Un Ashvamedha athénien?}

Je vais maintenant présenter une hypothèse selon laquelle les dysfonctions signalées s'expliquent si l'on considère qu'elles sont construites à partir de la réalisation aberrante du sacrifice du cheval indo-européen. Dans la mesure où ce rite exalte le pouvoir d'un roi en exercice, l'inversion ou l'altération de ses contenus dans le mythe athénien qui nous intéresse fournit une bonne explication de la fin de la dynastie Codride à Athènes.

G. Dumézil a expliqué le sens du rite : il compare l'Ashvamedha indien à l'October equus romain ${ }^{27}$. De plus, dans le monde celtique, il existe aussi un sacrifice du cheval lié à l'intronisation du roi, sacrifice qui s'apparente aux précédents tout en présentant des différences significatives ${ }^{28}$.

En Inde, il s'agit d'un sacrifice de guerriers réalisé pour le meilleur d'entre eux, le roi. La victime est le cheval situé à droite dans l'attelage vainqueur d'une course. Avant le sacrifice, on le laissait libre pendant un an sous la protection d'une escorte destinée à le défendre des ennemis du roi. S'il revenait sain et sauf, on le sacrifiait selon un rituel très complexe durant lequel son corps était divisé en trois et où l'on immolait des animaux d'autres espèces ${ }^{29}$. À Rome, le Flamen martialis sacrifiait le cheval situé à droite dans l'attelage de la bige qui avait vaincu dans une course de chars et on en séparait la tête et la queue. On emportait rapidement celle-ci à la Regia, un temple qui était l'ancienne résidence des rois, où l'on faisait goutter son sang sur un autel, alors que la tête faisait l'objet

27 DUMÉZIL, Religion, op. cit. (n. 15), 225-239; ID, Fêtes romaines d'été et d'automne, Paris, $1986^{2}$, p. 109-219. Avec une autre méthodologie et plus intéressé par les mythes, voir O'FLAHERTY, op. cit. (n. 4), p. 149-212.

28 J. PUHVEL, dans Vedic âsbvamedba- and Gaulish IIPOMIIDVOS, in Language, 31 (1955), p. 353354 , souligne l'homologie entre les termes védique (présent aussi comme nom d'un prince dans Rig Veda, V, 28, 4) et gaulois. Il met en évidence l'identité entre Asbva- et *Epo- (>IIPO) = cheval et

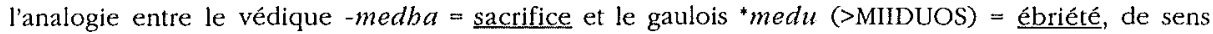
différent, mais proche du terme védique dont il est presque homophone. Notre Hippoménès pourrait être la version grecque de la série grâce à la transformation par métaphore du deuxième terme du mot composé. Celui-ci évoque, en védique, la boisson qui rend ivre et le sacrifice, mots qui ont la même racine, alors que le terme grec passe d'un sacrifice non réalisé, à une « force », ménos, ou à une folie, mania, toutes deux irrationnelles. F. LE ROUX, Recberches sur les éléments rituels d'élection royale irlandaise et celtique, in Ogam, 15 (1963), p. 123-137, rejette l'analogie entre l'épisode obscène de l'ashvamedha et le rite celtique de sacrifice d'une jument lors de l'intronisation d'un roi. Voir de plus DuMÉzIL, Fêtes, op. cit. (n. 27), p. 211-219. Le sacrifice lusitanien du cheval peut aussi être inclus dans l'héritage indo-européen, voir M.V. GARCíA QUINTELA, El Sacrificio Lusitano. Estudio Comparativo, in Latomus, 51/2 (1991), p. 337-354. M. CAPOZZA, art. cit. (n. 19), recueille un abondant matériel comparatif qui montre, surtout, les diverses fins et occasions des sacrifices de chevaux chez plusieurs peuples. Voir aussi la synthèse récente de E.C. POLOMÉ, Das Pferd in der Religion der eurasischen Völker et V. HAAS, Das Pferd in der betbitischen religiösen Überlieferung, tous deux dans B. HÄNSEL et S. ZIMMER (éds), Die Indogermanen und das Pferd, Budapest, 1994, p. 43-52 et 77-90 respectivement.

29 Les textes sont recueillis dans une traduction française par P.E. DUMONT, L'Asbvamedba. Description du sacrifice solennel du cheval dans le culte védique d'après les textes du Yajurveda blanc, Louvain, 1927. 
d'une dispute entre les habitants de deux quartiers. Si ceux de Subure gagnaient, ils la déposaient dans la Tour Mamilia, si la tête revenait à ceux de la Sacra Via, ils l'attachaient au mur de la Regia ${ }^{30}$.

Arrêtons-nous maintenant sur l'épisode obscène du rite védique qui n'a pas de parallèle dans le rituel romain. Il est représenté par les quatre épouses du roi : la mabisi, première épouse et reine consacrée accompagnée de filles de rois; la vavata ou favorite suivie de filles de familles de guerriers; la parivrkta ou la dédaignée accompagnée de filles de vaisya du plus haut statut et la palagali suivie de filles de familles de castes inférieures ${ }^{31}$. Ainsi ces femmes et leurs compagnes représentaient toute la société. Mais alors que la mabisi représentait un simulacre de rapport sexuel avec le cheval encore chaud que l'on venait de sacrifier, les autres se limitaient à échanger des obscénités avec les prêtres qui participaient à la cérémonie. Ce moment du rite avait pour but d'assurer la fécondité des participantes.

Pendant que la mabisi exécute le simulacre d'union narré de façon explicite $^{32}$, le roi s'adresse au cheval en s'identifiant sexuellement à lui lorsqu'il dit «mets dans la guda (vulve) de celle qui écarte les cuisses et fais entrer, ô mâle, celui qui oint, la grande joie des femmes ». Cette intervention est due à la «peur qu'il n'y ait quelqu'un qui rivalise avec le sacrifiant ${ }^{33}$, seul le roi ou le cheval dans cette situation particulière peuvent avoir des rapport sexuels avec la mabisi. Ensuite, chaque femme échange des expressions ayant un sens sexuel clair avec l'un des prêtres qui participent à la cérémonie. Le texte offre des explications pour chaque geste et chaque mot. Ainsi, dans le simulacre de rapport entre le cheval et la jeune femme (qui rejoint alors les autres femmes), on explique que son vagin est le peuple et le membre du cheval le pouvoir royal ${ }^{34}$. La réponse que reçoit le prêtre de la quatrième épouse nous intéresse aussi en tant qu'expression de la proximité conceptuelle entre les aliments et le rapport sexuel sous-jacente dans tout l'épisode: «Quand la gazelle mange du blé, elle méprise le bétail que l'on nourrit »; l'explication est que le blé est le peuple, la gazelle le pouvoir royal, et le pouvoir royal s'alimente du peuple ${ }^{35}$.

Nous pourrions maintenant comparer le rite védique d'accroissement du pouvoir royal avec le mythe athénien à propos de la fin de la dynastie royale en présentant ce dernier comme une inversion consciente des éléments significatifs du premier :

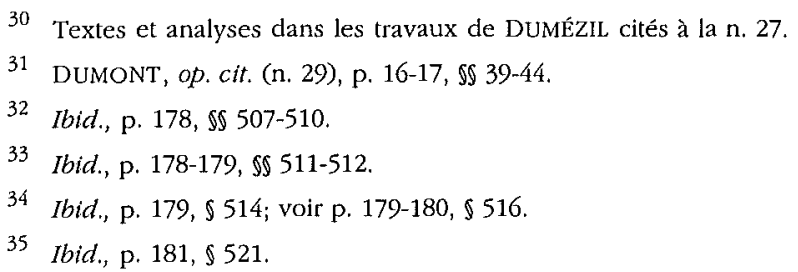




\begin{tabular}{|c|c|}
\hline Inde (rite) & Grèce (mythe) \\
\hline roi martial, dans la plénitude de son pouvoir & roi faible, efféminé \\
avec des épouses légitimes & rapport ambigu avec la fille/épouse \\
il tue le cheval martial & il tue le moichós attelé au char \\
sa mabisi s'unit au cheval $(=$ roi) & sa fille est mangée par le cheval (= roi / amant) \\
les femmes obtiennent la fécondité & la descendance est impossible \\
le roi voit croître son pouvoir & le roi perd son statut \\
\hline
\end{tabular}

Pour conclure, Hippoménès est un anti-guerrier qui prétend agir comme un super-guerrier. Au lieu de faire la guerre à l'extérieur, comme doit le faire un bon roi, il réalise une manifestation de force dans son entourage familial. Chacun de ses gestes rappelle une partie de l'ashvamedha, réalisé de manière aberrante, de telle sorte qu'en réalité il s'agit d'un anti-ashvamedha qui, au lieu d'augmenter sa force aux yeux des Athéniens en sa qualité de représentant de la vieille dynastie royale, provoque sa perte du pouvoir dans deux dimensions : d'une part il est destitué, comme l'affirme la tradition, d'autre part, cet acte annule ses possiblités d'avoir une descendance légitime ou de transmettre la royauté par l'intermédiaire de sa fille.

\section{Du mythe à l'histoire}

La dernière étape de notre analyse nous fait revenir du mythe à l'histoire. En effet, parmi les études indo-européennes, le cas grec a présenté des difficultés à cause des conditions spéciales de la présence d'éléments de l'héritage indoeuropéen clairement mis en valeur. Il n'en reste pas moins que l'on trouve de plus en plus d'éléments de cette sorte, bien qu'existe toujours la discussion sur la représentativité de chaque cas analysé ou sur la pertinence des comparaisons que l'on établit ${ }^{36}$.

En ce qui concerne le cas présent, il faut souligner que l'un des aspects où existe un degré élevé de conservatisme idéologique d'origine indo-européenne est précisément celui de la fonction royale. Ainsi F. Vian a dégagé une définition selon les trois fonctions de la Souveraineté dans différents épisodes mythiques ayant un lien avec la Béotie ${ }^{37}$. D'autre part, B. Sergent a étudié comment les

36 Par exemple, B. SERGENT, Les trois fonctions des Indo-européens dans la Grèce ancienne: Bilan critique, in Annales(ESC), 34 (1979), p. 1155-1186; D. PRALON, Le modèle triparti dans la philosopbie grecque du IVe siècle aC, in $A A V V$, Georges Dumézil, Paris, 1981, p. 121-136; G. DUMÉzIL, Apollon sonore, Paris, 1982.

37 F. VIAN, La triade des rols d'Orcomène; Étéoclès, Pblégyas, Minyas, in Hommages à G. Dumézil, Bruxelles, 1960, p. 215-224; ID., Les origines de Thèbes Cadmos et les Spartes, Paris, 1963 , p. $177-201$. 
attributs des rois des diverses dynasties royales spartiates correspondent de manière cohérente au plan Varuna ou Mitra de Souveraineté ${ }^{38}$.

De semblables approches ont été réalisées pour Athènes. Ainsi W.T. Magrath a examiné les figures de Cécrops, Érichthonios et Érechthée (les premiers rois de la première dynastie royale athénienne) et a montré le caractère royal (plan Varuna) du premier, sacerdotal (plan Mitra) du deuxième et clairement guerrier du troisième ${ }^{39}$. Pour sa part, R. Bodéüs a parcouru le chemin entre mythe et histoire pour analyser les figures institutionnelles des archontes athéniens, en mettant en rapport le basileus avec la royauté, le polémarque avec la guerre et l'archonte avec la fonction de reproduction sociale. Il a, de plus, cherché les vestiges d'une société athénienne organisée selon les trois fonctions qui pourrait servir de fondement au schéma institutionnel exposé ${ }^{40}$. A partir de ces résultats, la présence d'un élément de l'idéologie royale indo-européenne, en l'occurrence le sacrifice d'un cheval, ne devrait pas paraittre étrange. Il faut préciser que cet élément est modifié parce qu'il a pour fonction d'expliquer la fin d'une dynastie royale et non pas la gloire d'un roi, ce qui serait son objectif théorique.

Afin d'insister sur l'opportunité de l'hypothèse indo-européenne pour expliquer le cas d'Hippoménès, je vais suivre une autre voie. Du point de vue archéologique, Athènes et l'Attique en général sont des territoires où il n'y a pas eu, ou très peu, de destructions à la fin de l'époque mycénienne et où l'on assiste avant d'autres régions à la récupération de la culture matérielle tout au long des siècles obscurs ${ }^{41}$. D'autre part, il faut rappeler l'importance qu'a, dans l'imaginaire collectif de la cité athénienne, le mythe de l'autochtone ${ }^{42}$. Bien que l'on ne puisse pas relier ces deux circonstances, il est vrai que toutes deux peuvent aider à la persistance et à l'emploi autonome de traditions très anciennes particulièrement faciles à conserver dans un contexte où il existe une continuité de population et de société, et où l'idéologie officielle souligne, précisément, cette continuité.

\footnotetext{
38 B. SERGENT, La représentation spartiate de la royauté, in RHR, 189 (1976), p. 3-52.

39 W.T. MAGRATH, The Atbenian King-List and Indo-European Trifunctionality, in JIES, 3 (1975) p. $173-194$.

40 R. BODÉÜS, Société athénienne, sagesse grecque et idéal indo-européen, in AC, 41 (1972), p. 453-486.

41 A.M. SNODGRASS, The Dark Age of Greece, Edimburgh, 1971, p. 68 et passim; J.N. COLDSTREAM, Geometric Greece, Londres, 1977, p. 55-63, 73-81, 109-139; J. WHITLEY, Style and Society in Dark Age Greece. The changing face of a pre-literate society 1100-700 BC, Cambridge, 1991, centré sur l'Attique.

42 N. LORAUX, Les enfants d'Atbéna, Paris, 1984; E. MONTANARI, Il mito dell'autoctonia, Rome, 1981; d'un autre point de vue, l'affrontement lointain entre Athéniens et Pélasges souligne la présence constante des premiers dans l'Attique, voir HDT., VI, 136-139 et D. BRIQUEL, Les Pélasges en Italie, Paris-Rome, 1984, p. 285-293 à propos des traditions sur les Pélasges à Athènes.
} 
Pour en finir, il convient de citer une découverte archéologique qu'il est, comme presque toujours, difficile d'interpréter et encore plus difficile de mettre en rapport avec d'autres faits étrangers au domaine purement archéologique. Il s'agit d'une tholos situé à Acharnes en Crète (à $6 \mathrm{~km}$ au sud de Cnossos) datant du XIV e siècle av. J.-C. ${ }^{43}$. Y était enterrée une femme de haut rang, comme en témoigne la richesse du mobilier funéraire qui comptait des objets en métal noble et des pierres semi-précieuses. L'éditeur de cette découverte, J.A. Sakellarakis, n'hésite pas à considérer la défunte comme un membre de la famille royale (p. 156). Avec les restes de la femme, on a aussi trouvé ceux de divers animaux sacrifiés lors de la cérémonie funèbre. Il y avait parmi eux des boufs et l'auteur croit que ce sacrifice peut être relié à l'hypothèse de l'existence en Crète d'une royauté de caractère divin, mise en évidence par l'offrande de ces sacrifices à la reine morte. Mais ce qui nous intéresse ici, c'est la sépulture d'un cheval âgé de six ans, objet d'une manipulation rituelle complexe, durant laquelle on a séparé la tête du corps, la soumettant à un traitement particulier. C'est le premier cas d'inhumation d'un cheval en Crète. Il existe des parallèles en Grèce continentale, mais ces derniers ne sont pas reliés à une femme de rang royal comme à Acharnes ${ }^{44}$.

Comme nous le signalions plus haut, il est très difficile d'établir la relation entre un document archéologique et un mythe, et plus encore quand nous nous trouvons face à des cas exceptionnels ou sans parallèles évidents ni de l'un ni de l'autre. Cependant, nous ne pouvons manquer de souligner que l'association d'un cheval sacrifié avec une reine représente un parallèle rituel assez clair de ce qui, hypothétiquement, pourrait se trouver dans un endroit de l'Attique dénommé «Du cheval et de la Jeune Fille » qui fut considéré tardivement comme un sanctuaire (Dion Chr., XXXII, 78-79). Dans cette perspective, et à titre d'hypothèse car nous ne pouvons en dire plus, l'épisode d'Hippoménès pourrait être un aition certainement ancien (idée que défend Jacoby) à propos d'un lieu de sépulture du genre de celle qui a été mise au jour à Acharnes et dont le sens originel aurait été perdu. Cet aition aurait été revêtu de considérations politiques athéniennes en rapport avec la royauté dans lesquelles, comme nous l'avons vu, étaient présents sous différentes formes des éléments de l'héritage indoeuropéen et où, plus concrètement, on a eu recours au modèle d'un sacrifice du

43 J.A. SAKELLARAKIS, Das Kuppelgrab A von Achames und das kretiscb-mykeniscbe Tieropferritual, in Praebistorische Zeitscbrift, 45 (1970), p. 135-218.

44 Ibid., p. 199 et sq. Des découvertes plus récentes n'ajoutent pas de parallèles à ce cas, voir E. PROTONOTARIOU-DEILAKI, The tumuli of Mycenae and Dendra, in R. HÄGG, G.C. NORDQUIST (éds), Celebrations of Death and Divinity in the Bronze Age Argolid, Stockholm, 1990, p. 92-102 sur les tumulus de Dendra avec deux paires de chevaux d'attelage de chars de combat sans indication de la manière dont ils sont morts; apparemment il ne s'agit pas d'un sacrifice, comme dans le cas d'Acharnes. 
cheval en honneur du roi, réalisé de manière aberrante, pour expliquer la fin d'une dynastie royale et le sens oublié d'une ancienne tombe mycénienne ${ }^{45}$.

Departamento de Historia 1

Marco V. García Quintela

Facultade de Xeografía e Historia

Universidade de Santiago de Compostela

E - 15703 SANTIAGO DE COMPOSTELA

45 Ceci se fonde sur la réutilisation bien prouvée de tombes mycéniennes pendant la période de la Greek Renaissance, tombes transformées en sièges du récent culte au héros, COLDSTREAM, op. cit. (n. 41), p. 346-356 et fig. 110 avec carte de distribution du phénomène qui signale quatre gisements de l'Attique pour prouver cet usage de l'an 750 à l'an 650 (Eleusis, Menidi, Thorikos, Aliki) et à Athènes après l'an 650: ID., Hero Cults in the age of Homer, in JHS, 96 (1976), p. 8-17; A.M. SNODGRASS, Les onigines du culte des béros dans la Grèce antique, in G. GNOLI, J.-P. VERNANT (éds), La Mort, les morts, dans les sociétés anciennes, Cambridge-Paris, 1982, p. 107-119; T. HADZISTELIOU PRICE, Hero-Cult and Homer; in Historia, 22 (1973), p. 129-142; pour d'autres exemples récents voir P.G. CALligas, Hero-cult in Early Iron Age Greece, et V.K. LAMBriNoudaKIS, Veneration of ancestors in Geometric Naxos, in R. HÄGG, N. MARINATOS, G.C. NORDQUIST (éds), Early Greek Cult Practice, Stockholm, 1988, p. $229-34$ et 235-46. Soulignons que selon les chronographies anciennes Hippoménès se situerait entre les années 723 et 713 , ce qui coïncide avec les dates signalées plus haut. Voir aussi l'édition revue de F. DE POLIGNAC, La naissance de la cité grecque, Paris, 1995, p. $152-176$.

Je remercie Ana Iriarte et José Carlos Bermejo qui ont bien voulu lire ce texte, ainsi que Jordi Redondo qui m'a apporté son aide pour les questions philologiques. Traduction de l'espagnol de M.-P. Bouyssou. 